\title{
Study on Characteristics and Development of Xinjiang Makit Daolang Culture
}

\author{
Caixia Wang ${ }^{1, a}$, Chuanhui Zhang ${ }^{2, b}$ \\ ${ }^{1}$ College of Economics and Management, Tarim University, Alar, XinJiang, 843300 \\ ${ }^{a}$ email, ${ }^{b}$ email,
}

Keywords: Xinjiang Makit, Daolang Culture, Features, Development Method

\begin{abstract}
As a unique national culture, Daolang Xinjiang Uygur culture has a very important significance for the integrity of the constitution of our national culture. Makit County, Xinjiang is known as "Daolang town" title, years of cultural heritage and development of Daolang Makit County well preserved under the local customs cultures in the past. In recent years, more and more attention Daolang national cultural heritage and development, and is committed to Daolang as a cultural industry development in Xinjiang Maigaitite some culture. But also to develop better Daolang culture, paper characteristics and development of Xinjiang Makit Daolang culture were studied. The paper is divided into five parts: The first part outlines the development introduction background Makit Daolang culture; the second part It tells the story of the main manifestations Makit Daolang culture; the third part analyzes the Daolang cultural characteristics; fourth part gives some Makit Daolang cultural approach to development; part V Conclusion of paper summarized.
\end{abstract}

\section{Introduction}

Daolang peasant paintings. Daolang peasant paintings called "Daolang Videos" Swordsman these farmers painted on Daolang's home roof, walls, doors, windows and closets can be seen everywhere. Daolang peasant paintings mainly showed Makit local flavor, reflecting national characteristics, its mainly desert Populus, Bazaar crowd, donkey and Makit real life mosque, roasted Nang, etc. for the creation of content. Daolang farmers' paintings in color does not stick to the main conditions of the inherent color or color, with bright exaggerated, extravagant use of color, which allows farmers Videos Daolang formed a strong and distinctive color contrast, showed Daolang people natural, simple, bold personality. In fact, Daolang Daolang peasant paintings mainly reflects people's simple life, showing Daolang people with nature and, for effortless, highly personalized character and inclination, is a manifestation of national character.

Daolang Muqam. Speaking Swordsman culture, it certainly missed Muqam, Muqam also known as "Okuma." Twelve Uygur Muqam, it is actually Uygur music, folklore, dance, literature blend of an art form a whole, which Daolang Muqam is an important manifestation of the Twelve Muqam form. Daolang Muqam, mainly in Xinjiang Makit and Bachu, Awat, Shaya, Yuli and other endemic and dissemination, Daolang Muqam its odd unique artistic style has become the five kinds of Uygur Muqam geographical features and one of the most ethnic style Muqam, Daolang Muqam therefore also as "Daolang music" is one of the valuable cultural heritage of the Chinese nation is not indispensable.

Daolang Meshrep. Makit, entertainment rallies we tend to see people jumping Daolang group dance, which is called in the local Daolang Macey to just, Meshrep also called hot Macey \& P, with "place", "assembly" and "party" and other means, in fact, Makit one kind of dance will be happy expressions. Daolang Meshrep development is long, but it well preserved under the style and features of ancient Uygur dance of joy will be, it has become one of the national culture of Xinjiang very representative. Today, Daolang Meshrep this dance form and specific mass entertainment, and entertainment is no longer only a conventional meaning, as Sports people will give it a mental and physical health. 
Daolang Fangshenglingwu. In Daolang Meshrep we will find a very unique Daolang people jumping dance, which is a basic operation of the animal as mock objects Daolang dance, which we call the spirit of sacrifice imitation dance. Previously, the Daolang people, mainly in the Tarim Basin desert and jungle life, long-term hunting, farming for a living, and a variety of regular contact with animals as entertainment Daolang people will learn to get inspiration from the animal, created a Daolang person's unique spirit of sacrifice imitation dance. Over time, the spirit of sacrifice imitation dance spread to every corner of Xinjiang, Makit spread to become a major local dance features.

\section{The Main Features of Daolang Culture}

Regional Ethnic Features. Have to say, Makit Daolang culture is one of our great national culture of local ethnic characteristics, cultural Daolang well reflect a specific natural environment and human life custom case. It is because people Daolang unique life and lifestyle to ensure the area a unique Daolang culture, this unique cultural Daolang meet this local people's personality and customs, reflecting the strong national personality. Any culture, is a manifestation of character and personality, and it is this character and personality prompted the Daolang Muqam and Meshrep spread so far, the country has become very regional ethnic characteristics of the national culture.

Characteristics of song and dance entertainment. From the Daolang Muqam, Meshrep and imitation of animals, we can see the spirit of dance Daolang people and the pursuit of individuality and heroic characteristics. The three main forms Daolang culture reflects the characteristics of song and dance entertainment culture Daolang, Daolang who love music, love dance, love the entertainment they have no modification showed characteristic Daolang dance entertainment culture. After thousands of years of effort and accumulate it gives birth to now have this dance entertainment features Daolang culture, it not only Uighur spiritual wealth, but also the Chinese culture should not lose national culture.

More than yuan one Features. Daolang culture is actually not a single national culture, it is by the Mongols, Uighurs and local indigenous, etc. After years of fusion from one kind of multi ethnic culture, but it is also a branch of the Uighur ethnic culture. Cultural diversity refers to the so-called Daolang also retains the cultural heritage and customs of the Mongols, Uighurs and other ethnic and indigenous, plus, since Daolang living environment and lifestyle diversity, which presents cultural pluralism let Daolang of properties; one refers to the so-called Daolang after years of development in cultural heritage and gradually formed integrally with the Uighur culture, it has become a branch of Uighur culture. At the same time, precisely because Daolang cultural diversity, we can better retain the multi-ethnic culture, cultural practices, cultural unity Daolang also allows us an easier cultural heritage of this nation.

\section{Makit Daolang cultural development ideas}

Development of special industries have Daolang Culture. Construction of cultural industries with local characteristics of brand development for culture has insisted important development significance. Therefore, we can increase the development of Daolang cultural industry projects, such as construction has Daolang style building; the development of catering Daolang flavor; development Daolang clothing production and sales; conduct Daolang culture and tourism, and strive to build a culture Makit Daolang the special industries. Government should increase Makit Daolang leading backbone enterprise culture, historical and cultural resources of small-scale cultural industries, low utilization, investment and financing channels narrow efforts to support enterprises, with the development of multi-faceted security culture Daolang specialty industries. In short, it is to make a variety of industries Daolang culture and common development and achieve brand development Makit Daolang culture.

Daolang culture and tourism industry convergence. For a strange culture, people often are curious to experience psychological watch, therefore, Makit County Daolang cultural integration into the tourism industry will be an important way to take advantage of the unique regional culture 
Daolang national characteristics, tourism to attract more tourists, so not only can promote the development of tourism Makit, Daolang also better to develop their own culture. For example, to create a culture of live performance Daolang, let tourists to travel to appreciate the peculiar Daolang culture through performances; to be held Daolang Cultural Tourism Festival in Makit, attract more tourism enthusiasts; you can also carry out Daolang cultural experience tourism, travel through the experience to make travel enthusiasts Daolang cultural awareness, stimulate their desire for Daolang culture.

The introduction of modern teaching culture Daolang. Daolang from expressions of culture we see, Daolang culture is the cultural characteristics set painting, music, dance and entertainment in one of the diversity and unity, therefore, will Daolang culture into their teaching will have important pedagogical implications. For example, as one of the Daolang peasant paintings painting art teaching content; the Daolang Muqam introduced to music learning; spirit of sacrifice part of the dance as dance imitation learning; the Daolang culture to literature classroom learning. In short, by Daolang culture introduced to modern teaching, so that a new generation of young people about the culture and in-depth Daolang Daolang culture, such Daolang cultural development will naturally be less.

Using the media to expand its influence Daolang Culture. Media set sound, graphics, text in one, has a very important role in the spread, therefore, Daolang culture through the media will have a very important role in cultural development Daolang. For example, in recording people's lives Daolang Daolang cultural practices real program; formation Daolang cultural dance groups in television dance program; it has introduced gatherings Daolang culture in variety shows and so on. Such spread through television media, allowing more viewers Daolang cultural awareness and understanding, and let Daolang cultural impact more people.

Construction Daolang cultural propaganda website. With the popularity of the Internet, more and more people through the Internet to learn about some of their own cultural knowledge and did not know, therefore, in order to allow better hair Makit Daolang cultural development, can promote a culture of construction Daolang Website Daolang has a culture of detail, you can also record some video in the above reaction Makit's Daolang cultural practices, can also be used to promote its Makit some Daolang on culture and Tourism Festival, tourist attractions, so that more people can through the Internet You will be able to know and understand and Makit Makit Daolang culture.

To create a modern cultural and sports performances Daolang. Daolang build a modern cultural and sports performances, it refers to Daolang culture into modern sports and fitness among cultural performances. No external physical fitness is through exercise and physical fitness dance, dance is a spiritual sacrifice imitation imitating animal movements of dance, has a very important role in the physical, it can create Daolang cultural and sports fitness programs, and secondly, you can also set up cultural dance Daolang groups and music groups to perform, one to increase the diversity of modern dance, and secondly to Daolang cultural development has an important initiative.

\section{Conclusion}

Daolang Xinjiang Uygur culture as a special region national culture, national culture is one of the indispensable nation. Makit Daolang culture as the birthplace of the well preserved cultural practices, and by Daolang peasant paintings, Maqam, Meshrep and imitation sacrifice spiritual dance well reflect regional culture Daolang Nationality, song and dance entertainment , pluralistic one of the characteristics. In order to better carry forward the heritage and culture and development Makit Daolang Daolang culture, we need to adopt very Daolang development of cultural characteristic industry; Daolang industrial integration of culture and tourism development; Daolang will introduce modern teaching culture; to expand the use of media culture Daolang impact; Daolang construction sites and promote its culture to create a modern cultural and sports performances Daolang Daolang way to promote cultural development. China is a multi-ethnic country, a part of any national culture we are not missing. 


\section{References}

[1] Bi Jian. From the edge to the center --- Daolang cultural Development of Special Economic Zones under Kashi background [J]. Northern University for Nationalities (Philosophy and Social Science), 2010 (05).

[2] Li Yiheng. Xinjiang Daolang culture and its characteristics. Art under National Perspective [J]. Realistically, 2012 (04).

[3] Wang Xin, Wang Qiang. Daolang Dance Sports Culture Perspective [J]. Physical space, 2011 (01).

[4] Lin Miaoting. Daolang Xinjiang Intangible Cultural Heritage Tourism Network platform design [D]. Peking University, 2014.

[5] Zhang Tao. Xinjiang dance Daolang Meshrep Health Effects Ethnological Interpretation [J]. Sports Culture Guide, 2007 (03).

[6] Bao Bingfeng. culture and charm limiting factor in the development of Fang. Probe into Xinjiang Makit Daolang peasant painting [J]. Journal of Tarim University, 2010 (02).

[7] Zhou Ji. A variety of performances and symbiotic weeks Kyrgyzstan. Daolang Muqam recreational activities [J]. Journal of Xinjiang Arts Institute, 2004 (02).

[8] Amat river•Aikebaier. Daolang Xinjiang Makit County peasant paintings and heritage development dilemma analysis [J]. National Yilin, 2013 (03). 